\title{
Effect of Linear and Non-linear Resistance Exercise on Anaerobic Performance among Young Women
}

\author{
Homa Esmaeili \\ Department of sport Science, Shomal University, Iran \\ Ali Reza Amani (Corresponding author) \\ Department of sport Science, Shomal University, Iran \\ E-mail: a.amani@yahoo.com \\ Taher Afsharnezhad \\ Department of sport Science, Shomal University, Iran
}

Received: 21-05- 2015

doi:10.7575/aiac.ijkss.v.3n.3p.24
Accepted: 24-07- 2015

Published: 31-07- 2015

URL: http://dx.doi.org/10.7575/aiac.ijkss.v.3n.3p.24

\begin{abstract}
The main goals of strength training are improving muscle strength, power and muscle endurance. The objective of the current study is to compare two popular linear and nonlinear resistance exercises interventions on the anaerobic power. Previous research has shown differences intervention by the linear and non-linear resistance exercise in performance and strength in male athletes. By the way there are not enough data regarding female subjects. Eighteen young women subjects participated in this study. The subjects were randomly divided in two linear $(n=8)$ and nonlinear $(n=10)$ groups. An 11-week exercise protocol was applied in both groups. The anaerobic power and the body composition variables were measured in the pre and posttests. The results of this research substantiated that there was no difference in the peak anaerobic power between the linear and nonlinear models of the resistance exercise. The results of the current study proved greater improvement in the nonlinear model in contrast to the linear one in two groups at the final phase of the exercise $[\mathrm{F}(1,16)=6.270, \mathrm{p}=0.023]$. Greater improvement of the anaerobic power in the undulating group may have been influenced by less overtraining risk in the nonlinear model. The increase of the muscle and lean body mass (LBM) directly improved the anaerobic power by increasing the Atp-pc energy system. It was also observed that the nonlinear model of the resistance exercise experienced noticeable improvement in the body composition. Both linear and nonlinear models of resistance exercise improve anaerobic power, but at difference's situation coach and researcher should choose the best method according to type of sport and competition's season.
\end{abstract}

Keywords: Anaerobic power, Energy system, resistance exercise, performance

\section{Introduction}

The energy system in human body helps the cells to provide energy in different types of physical workouts and daily activities (Kenney, Wilmore, and Costill, 2015). Based on types of physical activities according to the amount and time of the energy needed, the different energy systems are recruited by the human bodies (Ekelund et al., 2002). ATP-Pcr, Aerobic and Lactic Acid are three of main energy systems in human bodies which differ from each other. The ATP-Pcr or at the other hand, Alactic energy system using on explosive situation and high speed performance such as 100 dash, weight lifting and shot put (Williams, 1999). The anaerobic power in human is closely related to the Atp-pc energy system. The anaerobic power is the amount of energy stored in the muscle which can used without oxygen (Margaria, Aghemo, and Rovelli, 1966). There are several factors that may affect the anaerobic power and performance in athletes. It is necessary to notice that the type of muscle fiber, amounts of the creatine storage in the muscle cells, the enzyme conditions and the muscle mass are among the most important variables which influence the anaerobic power (Demant and Rhodes, 1999; Volek and Kraemer, 1996). Additional research has shown that the resistance exercise may improve the anaerobic power by increasing the lean muscle mass and the creatine concentration on the cells (Chromiak et al., 2004; Docherty, Wenger, and Collis, 1987). However it has been shown that improving body composition will improve human performance and there is strong relationship between body composition and athletes performance (Amani, Somchit, Konting, \& Kok, 2010). The resistance exercise improves the fiber diameter and the cytosole volume of the intra cellular water (Paavolainen, Häkkinen, Hämäläinen, Nummela, and Rusko, 1999).

Nowadays, several resistance exercise strategies and systems help the coaches and athletes to achieve their peak of performance in competitions. There is a plethora of research reporting the positive effect of resistance exercise on the anaerobic power and anaerobic performance. Moreover, some of these systems and strategies have been reported to be more effective than the others. Linear and nonlinear models of resistance exercise have been employed recently by athletes and coaches (Bradley-Popovich, 2001). The linear model of periodization is more popular in sports but recent 
reports have proved the effectiveness of the nonlinear model of resistance exercise in some situations and sports. i.e. the researchers have demonstrated that the nonlinear exercise is more effective when the athletes do not have much more time or is useful during completion seasons as well as some commotions throughout the year (Fleck and Kraemer, 2014). The classic linear model generally takes a direct approach in which the training volume is steadily minimized while the intensity is progressively elevated until the competition moment. In contrast, the nonlinear or "undulating" model is characterized, among the other variables, by daily or microcycle (weekly) variations (Marques, 2005). It has been found out that periodized resistance training boosts the power of explosive performance in the final steps (the power and plyometric phases) (Newton and Kraemer, 1994). Some researchers compared two types of linear and nonlinear models of resistance training in the sport performance. Moreover, linear and nonlinear models of resistance exercise have been investigated in tennis, indicating that the nonlinear model of exercise could have better training theory for strength and conditioning because of avoiding the overtraining (Marques, 2005). The effect of linear and undulating models of resistance exercise on anthropometrical variables, Strength, and specific performance in judo was investigated in a study by Emerson. This research reported that both of the models improved the anthropometrical and body compositions variables in the same fashion. This study also indicated that there were no significant differences between the two groups in strength and performance variables (Franchini et al., 2014).

Nonetheless, several researches have reported contradictory results in the performance effect by these two types of resistance exercise theory. Hoffman and colleagues have illuminated that the linear model could be more effective in improving 1RM in freshman football players in comparison with the nonlinear model (Hoffman, Wendell, Cooper, and Kang, 2003). It has been reported that the anaerobic power is closely related to the muscle thickness and the muscle mass (Mercier, Mercier, Granier, Le Gallais, and Préfaut, 1992). In a study by Simao and colleagues has been reported greater muscle thickness and 1RM on the athletes undergoing the nonlinear resistance exercise (Simão et al., 2012).

The comparison between the linear and nonlinear resistance exercises requires more research and discussion regarding the variables in different situations and populations. Yet, the positive effect of each of these exercise strategies is still vague. One of most important aspects is investigating the effect of these exercises on the body composition, strength, power, and performance among athletes and non-athletes women. There is a paucity of literature regarding the linear and nonlinear resistance exercise program interventions among the women. Nowadays, most fitness and professional gyms use traditional and linear periodization programs for their athletes. Improving the data in comparing these two exercise strategies will help the coaches to choose the best way to achieve promising results. The purpose of the current study is to compare the effects of linear and nonlinear resistance exercise model interventions on the anaerobic power and anaerobic capacity of young women athletes.

\section{Method}

\subsection{Participants}

Eighteen young female athlete subjects with minimum 2 years of regular exercise and physical activities that had already enrolled in the exercise program were randomly recruited in two linear ( $\mathrm{n}=8$, age; 248.3 \pm 625.23 , weight; 59.30 \pm 8.12 , height; $160.625 \pm 6.523$ ) and nonlinear ( $\mathrm{n}=10$ age; $24.8 \pm 3.19$, weight; $50.98 \pm 6.725$, height; 161.4 \pm 2.451$)$ groups. The researchers in this study were limited in choosing the number of the subjects and all the subjects were volunteers and before starting the testing were asked to fill out a consent form. The participants were medically screened to prevent any risky condition during the experimental protocols. The screening was carried out for cardiovascular and pulmonary diseases, hypertension, orthopedic limitations to exercise, and renal disease.

\subsection{Anaerobic Performance and Test Protocol}

Wingate anaerobic protocol was used to determine the anaerobic power. The Wingate Anaerobic Test (WANT), developed at the Wingate Institute during the 1970s, is the most popular assessment tool to measure the peak anaerobic power, anaerobic fatigue, and the total anaerobic capacity. The Wingate test is most often carried out on a mechanically braked cycle ergometer or arm crank ergometer, for 30 seconds, at an "all out" pace. A counter is used to record revolutions of the flywheel for every 5-second intervals. Although the actual Wingate test is performed in a 30 -second time span, the subjects of this study were advised to complete a warm-up (3-5 minutes), followed by a recovery cool down (1-2 minutes). Resistance of the 0.075 of the body weight was added automatically to the flywheel after reaching the speed of 90rpm. The Monark Cycle device was calibrated prior to the test. The anthropometrical data were measured at the outset of the research. Pre and post-tests were applied before and after the exercise protocols. The subjects included in this research did not consume dietary supplements in the form of carbohydrates, proteins or amino acid and nor were any of them taking anabolic steroids (Shariat, Kargarfard, Danaee, and Tamrin, 2015).

\subsection{Exercise protocols}

A resistance exercise based on two linear and nonlinear strategies was applied for 11 weeks in 3 days/week. General preparation, hypertrophy, and the strength phase were applied for the linear model and general preparation and combination of hypertrophy and strength were used in the nonlinear model. For preventing injuries, general preparation was applied with the same volume and intensity in both groups in 3 weeks. The hypertrophy phase was applied with 9 sets a week with 6 training with $60-75 \%$ of 1RM for 4 weeks and the strength phase was applied with 6 sets a week with 6 training and 75 to $90 \%$ in 4 weeks (Fleck and Kraemer, 2014). 
To perform the squat jump, athletes were coached to align their feet shoulder width apart, while keeping their heels on the ground. They were instructed to bend their knees at 90 degrees and place hands on their hips to minimize counter movement (Van Soest and Bobbert, 1993; Rabahi et al., 2013). Then, athletes were asked to jump as high as possible (Rabahi et al., 2013). Two trials were performed in each test condition (self-focused, other-focused attention) for a total of four squat jumps.

\subsection{Data Analysis}

All the analyses of data were performed using the SPSS Statistical software (version 18). Mean and standard deviations were reported as the descriptive data. Analysis of variance with repeated measure was applied for determining the between and within groups. a t-student test was applied to determine the differences of the means and standard deviations in the per model from pre to post-test. Normality of the data was assessed by the Kolmogorov-Smirnov test. The Excel software was applied to demonstrate the graph. The statistical significance was set at $\mathrm{P}<0.05$.

\section{Results}

Analyzing the data showed that there was no significant differences between the two groups in the peak anaerobic power at $\mathrm{P}<0.05$ in 4 steps $\mathrm{F}(1,16)=2.263, \mathrm{p}=0.152$. analysis of variance in step one; $\mathrm{p}=0.298][\mathrm{F}(1,16)=1.155$, step two; $[\mathrm{F}(1,16)=0.014, \mathrm{p}=0.908]$, step three; $[\mathrm{F}(1,16)=2.784, \mathrm{p}=0.115]$ shown no significance difference in peak anaerobic power between groups and only the fourth step was significant difference in two groups $[\mathrm{F}(1,16)=6.270$, $\mathrm{p}=0.023]$. The statistical analysis revealed that there were significant differences between the peak anaerobic power from pre to post test in the non-linear model (pre-test; $(\mathrm{M}=191.53, \mathrm{SD}=82.34)$, post-test; $(\mathrm{M}=278.845, \mathrm{SD}=49.5)$. [T $(7)=2.707, p=0.030]$. Significant differences were observed between the peak anaerobic power from pre to post test in the nonlinear model (pre-test; $(\mathrm{M}=227.203, \mathrm{SD}=58.53)$, post-test; $(\mathrm{M}=340.26, \mathrm{SD}=53.32$. $[\mathrm{T}(9)=17.024, \mathrm{p}=0.000]$.

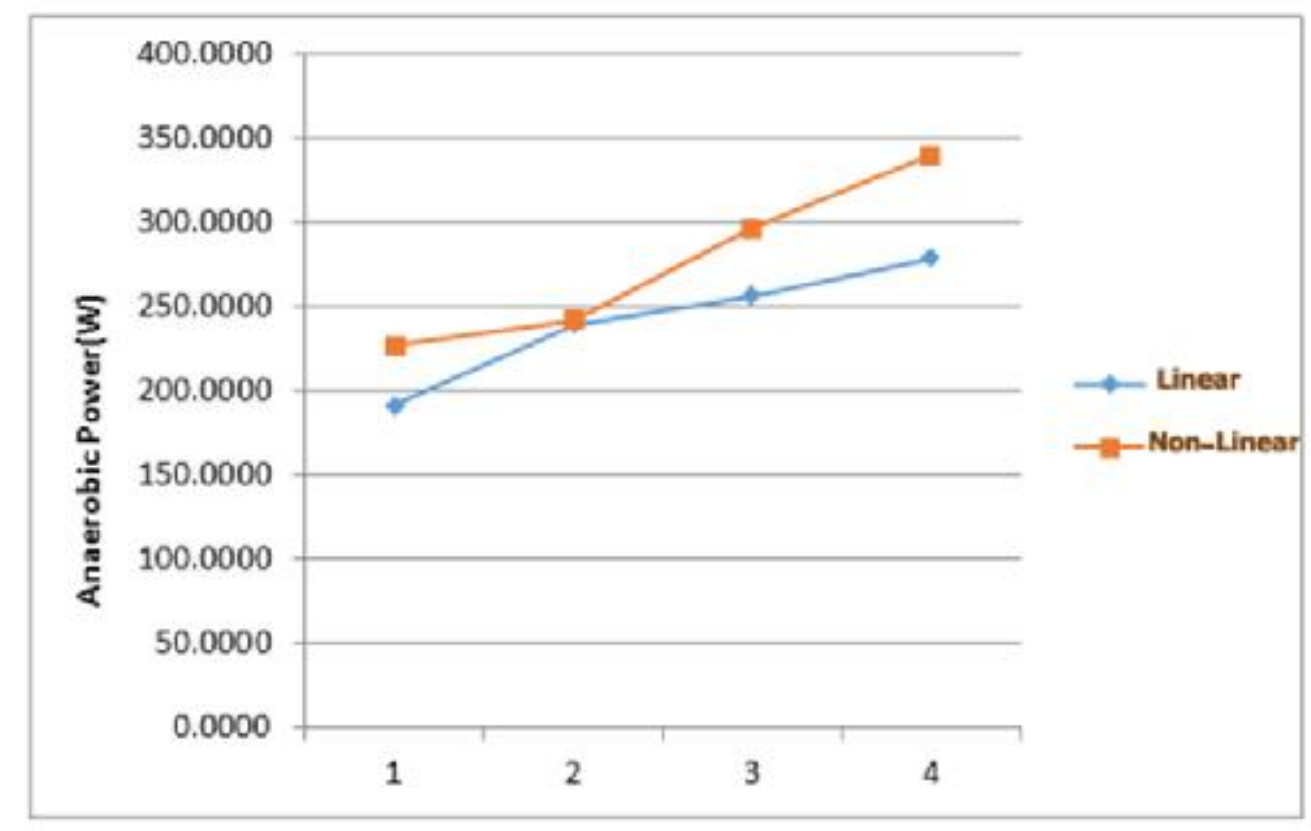

\section{Discussion and conclusion}

Nonlinear periodization in the resistance program is a more recent type of periodization in comparison with the linear model. A main goal in many linear training models is to peak strength and power immediately at the end of the peaking phase. The current study came up to a pattern for improving the anaerobic power in both linear and nonlinear groups. It needs to be asserted that slightly greater improvement has been reported in the nonlinear group compared to their counterparts (Fleck and Kraemer, 2014). The result of the current research is consistent with the recent findings (Franchini et al., 2014). The nonlinear model of resistance training may reduce the risk of overtraining in athletes during the competition and give more time for recovery in per week of workouts. In addition, greater body composition and anthropometrical improvement has been also reported (Chromiak et al., 2004) previously which may influence a greater anaerobic power and anaerobic performance in this research. Herrick and Stone have compared different exercise programs in young women, indicating that there is greater improvement in strength in the linear group (Herrick and Stone, 1996). The effect of the resistance exercise on the anaerobic power and the anaerobic performance are well reported in all the previous research. Nevertheless, achieving a high level of anaerobic performance in a short period of time in both women and men is still unknown and needs more discussion. The results of this study show that the power was improved in both groups. However, further studies are required to determine the reason of more improvement in the anaerobic power at the nonlinear model of the resistance training. The nonlinear model of the resistance exercise does not follow the standard pattern of intensity and volume of exercise and differences in the adaptation have been observed during the exercise program. After all, suggesting this type of exercise depends on numerous conditions and the nature of the sports. 


\section{References}

Amani, AR, Somchit, MN, Konting, Mohd Majid, \& Kok, Lian Yee. (2010). Relationship between body fat percent and maximal oxygen uptake among young adults. Journal of American Science, 6(4), 1-4.

Bradley-Popovich, Greg E. (2001). Nonlinear Versus Linear Periodization Models. Strength and Conditioning Journal, 23(1), 42.

Chromiak, Joseph A, Smedley, Brianne, Carpenter, William, Brown, Robert, Koh, Yun S, Lamberth, John G, Altorfer, Greg. (2004). Effect of a 10-week strength training program and recovery drink on body composition, muscular strength and endurance, and anaerobic power and capacity. Nutrition, 20(5), 420-427.

Demant, TW, and Rhodes, EC. (1999). Effects of creatine supplementation on exercise performance. Sports Medicine, 28(1), 49-60.

Docherty, D. A. V. I. D., Wenger, H. A., and Collis, M. L. (1987). The effects of resistance training on aerobic and anaerobic power of young boys. Medicine and science in sports and exercise, 19(4), 389-392.

Ekelund, Ulf, Åman, Jan, Yngve, Agneta, Renman, Cecilia, Westerterp, Klaas, and Sjöström, Michael. (2002). Physical activity but not energy expenditure is reduced in obese adolescents: a case-control study. The American journal of clinical nutrition, 76(5), 935-941.

Fleck, Steven J, and Kraemer, William. (2014). Designing Resistance Training Programs, 4E: Human Kinetics.

Franchini, E., Branco, B. M., Agostinho, M. F., Calmet, M., \& Candau, R. (2015). Influence of Linear and Undulating Strength Periodization on Physical Fitness, Physiological, and Performance Responses to Simulated Judo Matches. The Journal of Strength and Conditioning Research, 29(2), 358-367.

Herrick, Andrew B, and Stone, William J. (1996). The Effects of Periodization Versus Progressive Resistance Exercise on Upper and Lower Body Strength in Women. The Journal of Strength and Conditioning Research, 10(2), 72-76.

Hoffman, Jay R, Wendell, Michael, Cooper, Joshua, and Kang, Jie. (2003). Comparison between linear and nonlinear in-season training programs in freshman football players. The Journal of Strength and Conditioning Research, 17(3), $561-565$.

Kenney, W. L., Wilmore, J., \& Costill, D. (2015). Physiology of Sport and Exercise 6th Edition. Human kinetics.

Margaria, Rodolfo, Aghemo, P, and Rovelli, E. (1966). Measurement of muscular power (anaerobic) in man. Journal of Applied Physiology, 21(5), 1662-1664.

Marques, Mário A Cardoso. (2005). Strength Training in Adult Elite Tennis Players. Strength and Conditioning Journal, 27(5), 34-41.

Mercier, B, Mercier, J, Granier, P, Le Gallais, D, and Préfaut, Ch. (1992). Maximal anaerobic power: relationship to anthropometric characteristics during growth. International journal of sports medicine, 13(1), 21-26.

Newton, Robert U, and Kraemer, William J. (1994). Developing Explosive Muscular Power: Implications for a Mixed Methods Training Strategy. Strength and Conditioning Journal, 16(5), 20-31.

Paavolainen, Leena, Häkkinen, Keijo, Hämäläinen, Ismo, Nummela, Ari, and Rusko, Heikki. (1999). Explosivestrength training improves 5-km running time by improving running economy and muscle power. Journal of Applied Physiology, 86(5), 1527-1533.

Simão, R., Spineti, J., de Salles, B. F., Matta, T., Fernandes, L., Fleck, S. J., and Strom-Olsen, H. E. (2012). Comparison between nonlinear and linear periodized resistance training: hypertrophic and strength effects. The Journal of Strength \& Conditioning Research, 26(5), 1389-1395.

Shariat, A., Kargarfard, M., Danaee, M., and Tamrin, S. B. M. (2015). Intensive resistance exercise and circadian salivary testosterone concentrations among young male recreational lifters. The Journal of Strength \& Conditioning Research, 29(1), 151-158.

Volek, J. S., and Kraemer, W. J. (1996). Creatine supplementation: its effect on human muscular performance and body composition. The Journal of Strength and Conditioning Research, 10(3), 200-210.

Williams, Melvin H. (1999). Nutrition for health, fitness and sport: WCB/McGraw-Hill. 